\title{
La province au crible des rapports des commissaires départementaux du Directoire
}

Bernard Gainot

\section{(2) OpenEdition \\ 12 Journals}

Édition électronique

URL : https://journals.openedition.org/ahrf/791

DOI : 10.4000/ahrf.791

ISSN : 1952-403X

Éditeur :

Armand Colin, Société des études robespierristes

\section{Édition imprimée}

Date de publication : 1 décembre 2002

Pagination : 143-157

ISSN : 0003-4436

\section{Référence électronique}

Bernard Gainot, «La province au crible des rapports des commissaires départementaux du

Directoire ", Annales historiques de la Révolution française [En ligne], 330 | octobre-décembre 2002, mis en ligne le 17 avril 2008, consulté le 23 avril 2022. URL : http://journals.openedition.org/ahrf/791 ; DOI : https://doi.org/10.4000/ahrf.791 


\title{
LA PROVINCE AU CRIBLE DES RAPPORTS DES COMMISSAIRES DÉPARTEMENTAUX DU DIRECTOIRE
}

\author{
BERNARD GAINOT
}

\begin{abstract}
Sous le Directoire, les commissaires du pouvoir exécutif sont tenus de fournir des rapports, mensuels ou décadaires, à leurs autorités hiérarchiques. Les circulaires du ministère de l'Intérieur rappellent cette obligation, tout en normalisant les rubriques. Ainsi, une dizaine de rubriques fournissent les données économiques qui permettent au ministre de dresser le tableau de la République, tandis que la société locale est placée sous le regard permanent des autorités, qui établissent ainsi le baromètre de l'esprit public (surveillance des formes de déviance, adhésion aux rituels et symboles républicains, instruction publique).

Mais les réponses des commissaires départementaux aux sollicitations du pouvoir central sont variables, quoique de mieux en mieux rôdées au cours des années. On peut dresser une carte contrastée des départements producteurs d'informations et des départements silencieux, en émettant quelques hypothèses : préparation insuffisante à de lourdes tâches qui requièrent de plus en plus de professionnalisme, stratégies complexes déployées par les commissaires pour mieux asseoir leur pouvoir, à l'interface des réseaux locaux d'influence, et du dialogue avec un pouvoir central instable.

Ainsi, le rapport administratif, comme support du dialogue interne à la hiérarchie administrative, témoigne de la complexité des rapports Paris-province dans la période post-révolutionnaire, rapports qu'il serait extrêmement réducteur de traduire simplement en termes de centralisation.
\end{abstract}

Mots clés : commissaires centraux du pouvoir exécutif ; rapports décadaires ; esprit public ; centralisation ; données statistiques ; Directoire.

Cette communication s'inscrit dans un projet plus vaste, dont les grandes lignes ont été présentées par ailleurs (1). Commençons donc par rappeler l'objet et le cadre de notre enquête.

(1) Séminaire de l'Institut d'histoire de la Révolution française, 10 avril 1999; journée d'étude du laboratoire «collectivités locales » de l'Université d'Orléans, Bourges, 4 décembre 1999.

Annales historiques de la Révolution française - 2002 - $N^{\circ} 4$ [143 à 157] 
Le corpus est constitué d'environ trois cents commissaires nommés par le Directoire exécutif auprès des administrations départementales de quatre-vingt-dix-neuf départements, entre brumaire an IV (octobre 1795) et brumaire an VIII (novembre 1799).

Ils constituent un lien entre l'institution préfectorale, qui a été l'objet de plusieurs mises au point récentes à l'occasion de ses deux siècles d'existence (2), et les procureurs-généraux-syndics de département des premières années de la Révolution (3). Force est de constater que, si les préfets retiennent à juste titre l'attention, les procureurs-syndics et les commissaires du Directoire exécutif n'ont guère éveillé la curiosité de chercheurs autres que les érudits locaux. Il faut faire une exception pour Raymond Guyot (4) et, une fois de plus, pour Alphonse Aulard (5). L'un et l'autre abordent toutefois l'institution sous l'angle de la centralisation post-révolutionnaire. Selon Aulard, le commissaire est l'agent essentiel d'un processus administratif qui justifie pleinement son titre de commissaire « central » :

«Par l'institution des commissaires, par la marche de l'administration, par une tendance générale des esprits et des mœurs vers l'unité, non seulement le mouvement de décentralisation administrative qui avait paru se dessiner pendant la période thermidorienne s'arrêta, mais il se produisit une centralisation plus marquée encore que dans la période terroriste du gouvernement révolutionnaire, et qui alla en se fortifiant chaque jour davantage. »

Au début de l'an IV, tout comme pour la période comprise entre brumaire et ventôse de l'an VIII, se placent deux délicates périodes de transition : passage du gouvernement révolutionnaire au régime constitutionnel à l'automne 1795, réagencement de l'action des agents du pouvoir exécutif au début du Consulat. Ces périodes de transition sont déterminantes pour toute l'orientation future de l'institution, aussi bien sur le plan de la décision (politique), que sur celui de l'action (administrative).

Je m'attacherai plus particulièrement ici à montrer comment les problématiques retenues, circulation de l'information et prise de décision politique, peuvent renouveler les recherches sur l'administration locale, en l'occurrence sur l'administration des départements. Elles répondent beaucoup mieux à la réalité de la pratique administrative que les formulations classiques en termes de «centralisation ». Ces dernières présentent en effet l'inconvénient de figer une situation mouvante, dans laquelle s'enchevêtrent

(2) Signalons notamment le quatrième volume des journées d'études Du Directoire au Consulat (Jean-Pierre Jessenne, édit.), Centre de Recherche sur l'Histoire de l'Europe du Nord-Ouest, Rouen, 2001 ; et le $\mathrm{n}^{\circ}$ spécial des Annales de l'Est : « De l'intendant au préfet », 2,2000.

(3) Continuité soulignée par Alphonse AULARD, Histoire politique de la Révolution française; l'origine de la démocratie et de la République, 1789 - 1804, Paris, Armand Colin, 1926, p. 605.

(4) R. GUYOT, « Du Directoire au Consulat : les transitions », Revue historique, tome XI, 1912, pp. 1-30.

(5) A. AULARD, Histoire politique de la Révolution..., op. cit., p. 607. 
la personnalité des acteurs, leur représentation du réel, leur formation professionnelle, les réseaux de relations et d'influence, les dosages locaux de la coutume et de la loi, au bénéfice exclusif d'un modèle rigide et antinomique, en opposant ce qui vient d'en haut (la norme étatique), à ce qui résiste localement (que ce soit l'ébauche d'une société civile ou les communautés traditionnelles).

\section{Les instructions du pouvoir central.}

La source essentielle pour connaître la pratique administrative des commissaires, ce sont les comptes rendus périodiques de leur gestion. Ils sont partiellement rassemblés dans la sous-série FlcIII (Espritpublic et élections) des Archives nationales. Ces enquêtes, plus ou moins standardisées et ces rapports mensuels ou décadaires, renvoient d'abord au cadre réglementaire global fixé par plusieurs circulaires.

En effet, le dispositif constitutionnel était très vague :

« article 191 : Le Directoire exécutif nomme, auprès de chaque administration départementale et municipale, un commissaire qu'il révoque lorsqu'il le juge convenable. Ce commissaire surveille et requiert l'exécution des lois.

article 192 : Le commissaire près de chaque administration locale, doit être pris parmi les citoyens domiciliés depuis un an dans le département où cette administration est établie. Il doit être âgé de vingt-cinq ans au moins. » (6)

Aussi a-t-il été progressivement complété par une série de textes réglementaires, en commençant par le décret du 21 fructidor an III (7 septembre 1795) qui impose l'obligation de résidence du commissaire dans la localité même où se tiennent les séances de l'administration auprès de laquelle il est en fonction. C'est une disposition essentielle, qui fait du commissaire un homme de terrain, inséré dans le milieu local.

Les autres prescriptions du décret portent sur les traitements, et stipulent que, si le commissaire assiste aux délibérations de l'administration centrale du département, il n'a toutefois pas voix délibérative. C'est un rappel tout à fait significatif des principes de fonctionnement d'un régime véritablement représentatif.

Les instructions des ministres de l'Intérieur successifs vont définir les tâches pratiques des commissaires. Celles de Bénezech du 22 brumaire an IV (3 novembre 1795), puis de Barras (7) du 12 frimaire an IV

(6)J. GODECHOT, Les constitutions de la France depuis 1789, Paris, Garnier-Flammarion, 1979, pp. $122-123$.

(7) Signée Reubell, alors président du Directoire, elle serait en réalité de la main de Barras, selon Aulard. Mise au point qui est loin d'être purement formelle, quant au fonctionnement interne de l'Exécutif, que des recherches ultérieures pourraient permettre de clarifier. 
(3 décembre 1795) sont fondamentales. Elles définissent les missions du commissaire, tout en développant longuement l'esprit qui doit guider ces missions, qui sont conjointement des missions d'ordre public, et des missions d'esprit public, ce qui pose naturellement de délicats problèmes d'attribution de compétences et de coopération avec d'autres agents des ministères (police, armée par le biais de la gendarmerie).

L'instruction de brumaire an IV impose la rédaction d'un rapport administratif et définit les rubriques types qui le structurent; en premier lieu, la levée des contributions, puis la répression de la désertion et du brigandage, la surveillance des émigrés, la vie économique (confection et entretien des grandes routes, excavation des canaux de navigation, amélioration de l'agriculture, multiplication des bestiaux de toute espèce, établissements à créer, et plus encore secours nécessaires à l'entretien des établissements déjà formés), puis la surveillance des mœurs, la célébration des fêtes nationales, et enfin l'instruction publique.

La circulaire de frimaire an IV est encore plus explicite quant à la forme de ce rapport administratif, et quant à sa fréquence. Sont pareillement précisées les voies hiérarchiques qui lui conféreront son statut de document officiel :

«Le zèle, les lumières même, et le patriotisme ne suffisent point pour faire marcher la machine constitutionnelle; il faut que l'esprit d'ordre et de méthode utilise toutes ses bonnes qualités.

Il faut surtout conserver les distances et la hiérarchie des pouvoirs.

Chaque commissaire de canton devra donc correspondre directement, et au moins une fois chaque décade, avec le commissaire général du département.

Ils pourront, dans les cas pressants, dans les événements extraordinaires, écrire directement au ministre.

Chaque commissaire général correspondra journellement (8) avec le ministre de l'Intérieur, et lui donnera le dépouillement méthodique et substantiel des rapports qui lui auront été faits. Il y joindra ses observations, sa critique, ses vues et ses découvertes personnelles. »

La tonalité générale de la circulaire est imprégnée de l'esprit de " concentration républicaine », caractéristique des lendemains des journées de vendémiaire. Pour Barras (si tant est qu'il soit bien le concepteur du document, mais c'est fort plausible), il ne fait aucun doute que le royalisme est, sinon le seul, du moins le véritable ennemi de l'intérieur. De fait, la période du Premier Directoire (1795-1797) est généralement marquée au niveau départemental par la forte présence du commissaire, persuadé d'incarner tout ensemble la loi et la volonté de la République ; ce qui indispose

(8) Mot souligné dans le texte (réimpression de l'ancien Moniteur, tome XXVIII, 20 frimaire an IV 11 décembre 1795), p. 636. 
les administrateurs et les magistrats, bien vite soupçonnés de complaisance par le commissaire, sinon de complicité, avec les puissances qui encadraient la société d'Ancien Régime. Les conflits s'enveniment, particulièrement après les élections du printemps 1797. Le coup d'État «anti-royaliste »du 18 fructidor an $\mathrm{V}$ fut également la victoire du commissaire républicain sur l'administrateur enclin au compromis avec les forces sociales traditionnelles.

C'est dans ce contexte que paraît la troisième circulaire, signée par François de Neufchâteau, le 21 fructidor an V (7 septembre 1797). Il y constate que le travail exigé par les précédentes circulaires n'a pas été fait, de telle sorte que :

« des événements majeurs et caractéristiques ne venaient à ma connaissance que d'une manière indirecte, par les papiers publics ou des correspondants privés; d'autres, toujours tremblants de se voir compromis, n'osaient dire la vérité, ou exigeaient qu'on regardât les plus simples renseignements comme des confidences. » (9)

Après avoir attribué ces négligences, ou ce manque d'énergie, aux circonstances politiques (les entraves mises par les royalistes au fonctionnement du gouvernement républicain), le ministre définit par une métaphore les fonctions de ses agents :

«Souvenez-vous qu'un commissaire du Directoire exécutif doit être, en quelque sorte, une glace pure et fidèle où le gouvernement doit voir se réfléchir les objets tels qu'ils sont.. »

Puis, les cadres du travail administratif sont rappelés :

«Les commissaires des cantons doivent vous rendre compte, de décade en décade, de l'exécution des lois et de l'état de leur canton; mais, à votre tour, citoyens, vous devez m'envoyer un compte décadaire, formé non seulement du résultat de ceux qui vous sont adressés, mais de vos observations, et de vos notes particulières. »

La hiérarchie des rubriques est sensiblement bouleversée, l'esprit public ravissant la première place à la levée des contributions (10). Changement significatif...

La dernière circulaire d'application qui retiendra notre attention est celle du 23 prairial an VI (11 juin 1798), publiée neuf mois seulement après celle de François de Neufchâteau. Il faut croire que la collecte est encore

(9) Bulletin des lois, an V et AN, AD XIX A (140).

(10) Voici quel est l'ordre de présentation des rubriques dans la circulaire de François de Neufchâteau : esprit publique, instruction publique, police générale, récoltes et subsistances, police champêtre, police des cultes, hospices et établissements de bienfaisance, épidémies et épizooties, maisons d'arrêt, prisons, recouvrement des contributions, grandes routes et chemins vicinaux, agriculture et plantations, forêts, commerce et industrie, force armée, concours entre les diverses autorités constituées pour opérer la sûreté publique, la répression des délits, etc. 
bien loin de fonctionner régulièrement. Toutefois, son successeur, Letourneux, impute les retards constatés dans l'envoi des comptes rendus, non à l'oubli ou à la négligence, mais à la :

«trop fréquente rénovation d'un travail auquel vos autres attributions, maintenant très multipliées, ne vous permettraient pas de vous livrer à des époques si rapprochées.

J'ai obtenu, par ces considérations, l'autorisation de permettre que les comptes décadaires fussent réduits à un compte chaque mois; mais, en demandant cette facilité, j'ai cru pouvoir promettre, en votre nom, une précision méthodique, des résultats raisonnés, quelques vues neuves, surtout l'exactitude dans l'envoi. » (11)

La forme du compte rendu mensuel (et non plus décadaire) est à peu près fixée ; l'attention portée à la lourdeur du travail des commissaires est un indice de bonne qualité de la communication entre le ministre et ses agents (12). À la simplification des tâches administratives (accorder aux commissaires centraux une plus grande latitude pour la collecte des informations qui proviennent du niveau cantonal, assurer une plus grande standardisation des formulaires administratifs en généralisant la duplication de modèles, éviter les répétitions des observations portant, par exemple, sur des données économiques ou sur l'état de l'esprit public, relevées décade par décade), répond une efficacité certaine dans la collecte des renseignements. C'est en l'an VII que l'on enregistre proportionnellement le plus grand nombre de comptes rendus (13).

\section{Les comptes rendus des commissaires locaux.}

Pour apprécier la valeur du contenu de ces comptes rendus, certaines précautions méthodologiques s'imposent.

La théorie du commissaire, « reflet» de la réalité sociale, telle qu'elle est exposée par François de Neufchâteau, est une vue de l'esprit. L'exploitation des données fournies par les commissaires a bien souvent été entreprise sans aucun recul, dans le cadre notamment de la confection de monographies départementales. Selon cette perspective positiviste, le commissaire central se contenterait d'enregistrer les faits économiques (bonnes ou mauvaises récoltes, gel, épizooties, etc.), les faits sociaux et

(11) AN, F1a (23)

(12) Lauriane ALBRECHT, La correspondance administrative des autorités départementales avec le ministère de l'Intérieur sous le Directoire (1795-1799). Les comptes des administrations et des commissaires, mémoire de DEA d'histoire, sous la direction de Dominique Margairaz et Denis Woronoff, Université Paris I, juin 2000.

(13) Le mémoire de L. ALBRECHT, La correspondance..., op. cit., enregistre 458 envois pour l'an Vil, et 670 pour l'an VI : mais, en l'occurrence, ceux de l'an VI sont établis par décade, tandis que ceux de l'an VII, conformément à la circulaire de Letourneux, sont mensuels. 
mentaux que lui transmettent les commissaires cantonaux, ou qu'il a pu observer lui-même et les réticences des populations envers les cérémonies officielles : attachement au culte catholique et à l'observance du dimanche, obéissance aux consignes des prêtres réfractaires, pour nous en tenir à quelques exemples. Le ministre, et plus tard l'historien, coordonne tous ces faits pour en dresser un tableau synthétique. L'ambition globale et avouée était de parvenir à établir un tableau de la République.

Toutefois, cette ambition se heurte à de nombreuses difficultés ; lacunes de l'information, certes, mais pas seulement. Il faut aussi tenir compte des filtres socio-culturels (14) que le commissaire mobilise lors de son relevé du réel observable ; des stratégies mises en œuvre par ledit commissaire, afin de préserver ou de consolider la portion de pouvoir qu'il occupe. Il faut enfin s'interroger sur la signification même de l'opération intellectuelle de mise en tableau, et de la relation entre cette opération et l'état des sciences sociales (notamment) à la charnière des $\mathrm{XVIII}^{\mathrm{e}}$ et $\mathrm{XIX}^{\mathrm{e}}$ siècles (15).

\section{Les lacunes de l'information.}

Il y a de grandes disparités en ce qui concerne la mise en œuvre des recommandations contenues dans les circulaires ministérielles (16). Pour vingt départements qui ont fait parvenir au ministre plus de vingt comptes rendus (17) - dont six plus de trente (18) -, il y en a sept (19) qui n'ont fait parvenir aucun compte rendu sur les quatre années. De même, la fréquence varie énormément selon les périodes. Numériquement, c'est en l'an VI que les commissaires envoient le plus grand nombre de comptes rendus (six cent soixante-dix). Mais il faut tenir compte du fait que les comptes rendus sont alors décadaires ; à partir de l'an VII, ceux-ci deviennent mensuels. Proportionnellement, la fréquence est plus élevée (quatre cent cinquante-huit), et

(14) L'expression est empruntée à Stuart WOOLF, Napoléon et la conquête de l'Europe, Paris, Flammarion, collection « Histoires », 1990.

(15) Jean-Claude PERROT, L'âge d'or de la statistique régionale française (an IV-1804), Paris, Éditions de la Société des études robespierristes, 1977 ; Marie-Noëlle BOURGUET, Déchiffrer la France; la statistique départementale à l'époque napoléonienne, Paris, Éditions des archives contemporaines, 1988; Jean-Luc CHAPPEY, La Société des Observateurs de l'Homme (1799-1804), Paris, Société des études robespierristes, 2002; Dominique MARGAIRAZ, Du lieu commun à l'esprit public, François de Neufchâteau, essai de biographie intellectuelle, thèse d'État, Paris I, décembre 2001.

(16) Voir la carte de l'origine départementale des comptes rendus mensuels ou décadaires des commissaires, dressée à partir du dépouillement de la série FlcIII des Archives nationales, dans L. ALBRECHT, La correspondance..., op. cit.

(17) Dans l'ordre : Lot-et-Garonne (38), Pyrénées-Orientales (37), Ardèche (33), Puy-deDôme (32), Lozère (30), Hérault (30), Vendée (29), Haute-Saône (28), Creuse (28), Vienne (26), AlpesMaritimes (25), Landes (24), Meurthe (23), Morbihan (22), Cher (22), Aude (22), Eure-et-Loir (21), Nord (21), Isère (21), Loir-et-Cher (21).

(18) Ardèche, Lot-et-Garonne, Lozère, Pyrénées-Orientales, Puy-de-Dôme, Hérault.

(19) Bouches-du-Rhône, Charente-Inférieure, Côte-d'Or, Gard, Hautes-Alpes, Manche, Haute-Marne. 
la progression serait à peu près linéaire, puisque l'on n'enregistre que dix comptes rendus en l'an V, et vingt-cinq en l'an IV. Au fil des ans, les efforts porteraient donc leurs fruits, avec un seuil très net au lendemain du 18 fructidor. L'existence de ce seuil montre que les seules explications techniques, liées à la progressivité de la mise en place de l'institution, ne sauraient suffire. La pratique administrative est déterminée par les contraintes de l'espace-temps, essentielles pour évaluer la circulation de l'information. Pour l'époque napoléonienne, rien n'est changé par rapport à la seconde moitié du XVIII ${ }^{\mathrm{e}}$ siècle : il faut mettre l'accent sur la vitesse de circulation des courriers (20). Celle-ci relativise tous les jugements péremptoires portés sur la prétendue «centralisation administrative ». Le travail administratif s'insère dans une perception de l'espace déterminée par le clivage espace utile/espace vécu. L'espace utile, c'est celui des grandes routes, au long desquelles sont établis bureaux de poste et brigades de gendarmerie. Il faudrait bien sûr compter également les voies navigables sillonnées par les coches d'eau. C'est là le terrain d'intervention privilégié du commissaire central, et de ses interlocuteurs principaux (à titre d'agents de l'Exécutif, et non d'administrateurs élus) : le directeur des messageries et le lieutenant commandant la compagnie départementale de gendarmerie. Commissaires cantonaux et gendarmes des brigades locales doivent, tout autant que les administrateurs municipaux ou les juges de paix, davantage tenir compte de l'espace vécu, celui des travaux et des jours, des villages « quasi immobiles ». Ces deux espaces ne sont pas étanches; les courriers administratifs (les circulaires ministérielles exigent des commissaires cantonaux une correspondance régulière avec le commissaire central) font le lien. Mais le ministre exige un type de contact beaucoup plus direct avec le milieu local, de la part du commissaire central. Il doit effectuer régulièrement des visites, «qui évoquent les célèbres chevauchées des élus sous l'Ancien Régime », insiste François de Neufchâteau, redevenu ministre de l'Intérieur en septembre 1798 (21).

Bien entendu, qualité de l'information et contrôle de l'espace ne sont pas placés dans un rapport univoque. On peut même supposer que les zones départementales les moins faciles d'accès, notamment les zones montagneuses centrales, sont celles sur lesquelles la pression du ministère fut la plus forte pour obtenir des comptes rendus réguliers. Ainsi, parmi les départements les plus prolixes, pouvons-nous relever les régions périphériques du Massif Central (Creuse, Ardèche, Allier), voire du territoire national

(20) Atlas de la Révolution française, tome 1, «Routes et communications », sous la direction de Guy ARBELLOT et Bernard LEPETIT, Paris, Éditions de l'EHESS, 1987. Et aussi M.-N. BOURGUET, Déchiffrer la France..., op. cit. ; S. WOOLF, Napoléon et la conquête de l'Europe, op. cit.

(21) Circulaire du 27 fructidor an VI (13 septembre 1798), commentée par J.-C. PERROT, L'âge d'or de la statistique..., op. cit., p. 25. 
(Pyrénées-Orientales, Alpes-Maritimes), ou encore celles dont le loyalisme est douteux (Vendée).

La qualité de l'information peut également être corrélée à la stabilité de l'informateur. Plusieurs de ces départements prolixes ont le même informateur tout au long des quatre années d'existence de la République constitutionnelle, ou tout du moins un commissaire installé pendant un laps de temps suffisant pour un bon rodage de la technique du rapport administratif. Tel est le cas de Lafond-Cujula, en poste pendant quarante-deux mois dans le Lot-et-Garonne, de Vaquer en poste quarante-trois mois dans les Pyrénées-Orientales, de Braveix en poste quarante-deux mois dans l'Ardèche, de Boutarel (quarante et un mois dans le Puy-de-Dôme), de Servière (quarante et un mois en Lozère), de Coyaud (quarante-trois mois en Vendée), de Bonnefont (quarante mois dans la Vienne), ou de Michelet (quarante-sept mois dans la Creuse). Bien sûr, il y a des contre-exemples, le plus marquant étant celui de Bontous dans les Hautes-Alpes, qui reste quarante-sept mois au poste de commissaire. La famille Bontous contrôle le département, puisque le fils est la figure centrale de la députation au Corps législatif. Il en irait de même, toute proportion gardée, avec les Laloy dans la Haute-Marne (22).

Stabilité dans la fonction et pression ministérielle sont des données importantes pour rendre compte de la nature du travail administratif. Elles ne suffisent pas. La personnalité même du commissaire joue un rôle considérable : ses opinions politiques et ses compétences professionnelles, notamment.

\section{La personnalité des commissaires.}

Le regard porté par le commissaire sur son environnement socioculturel est doublement déterminé : tout d'abord, par la représentation qu'il a de celui-ci, mais également par le fait que, soumis à l'obligation de résidence, il en est partie prenante.

Globalement, le regard porté par le commissaire sur la société civile, à plus forte raison s'il a été, à un moment ou à un autre, acteur du processus révolutionnaire, est empreint de méfiance. Le projet de régénération sociale placé au cœur du projet révolutionnaire, implique, pour être mené à son terme, un arrachement du citoyen à ses attaches communautaires. Ce processus demande du temps; la société française de l'époque du Directoire

(22) Bernard GAINOT, «Propositions pour une enquête prosopographique sur les commissaires centraux de Directoire », laboratoire « collectivités locales » de l'Université d'Orléans, op. cit. En fait, si les comptes rendus du commissaire Laloy, le frère du représentant, ne se trouvent pas rassemblés dans la soussérie FlcIII, on les trouve dans la sous-série départementale AF III 246. 
est encore régie par des rapports coutumiers séculaires, un ensemble complexe de liens de dépendance et de fidélités hiérarchiques.

La méfiance peut conduire à des tensions persistantes, voire à des comportements vite dénoncés comme «tyranniques » par les adversaires. Le commissaire central du département de Saône-et-Loire en l'an VII, Nicolas Carteron, est un esprit inquiet, que traduisent ses rapports :

« Il est enclin à toujours voir des "égorgeurs" à côté de lui, et les troupes ennemies prêtes à entrer en France. Tout ce qui n'est pas aussi pétulant que lui est contre-révolutionnaire, et la crainte où il est de voir revenir l'Ancien Régime fait que, souvent, il n'épargne pas dans ses transports les meilleurs citoyens. » (23)

Ce comportement inquiet conduit à des rapports difficiles avec les administrés ; il est souvent présenté par ses adversaires comme caractéristique de celui des commissaires de sensibilité ultra-républicaine, jacobine.

À l'inverse, d'autres commissaires sont enclins au compromis. Dans leurs rapports, leur attention se porte plutôt vers la rentrée des contributions publiques, l'entretien des routes, l'organisation formelle des cérémonies publiques. La préoccupation première est la conciliation entre ordre public et esprit public, ce qui implique un mode de gestion du conflit local fondé sur le compromis avec les cadres sociaux traditionnels, sur la transaction. Compromis que leurs adversaires assimilent bien vite à la compromission avec les ennemis de l'État républicain, voire à une complicité avec les fauteurs de troubles et les brigands. Néanmoins, dans la conjoncture précise de fructidor an V, la ligne de partage entre les deux attitudes passe plutôt entre les commissaires, garants de la loi républicaine, et les administrateurs élus, complices des pouvoirs locaux, ce qui pose la délicate question du rapport entre la souveraineté de la loi et la légitimité du régime républicain. Sous le Second Directoire, les commissaires sont à leur tour accusés d'interventionnisme par les groupes politiques républicains, qui les avaient dans l'ensemble soutenus dans leur lutte contre les administrations locales. Les analyses du contexte électoral en l'an VI, et plus encore en l'an VII, montrent comment le commissaire est chargé par le ministre de l'Intérieur de préparer les élections, de faire pression sur les citoyens, de se présenter comme le «candidat officiel » du gouvernement.

La rédaction et la fréquence des comptes rendus s'inscrivent alors dans des stratégies de pouvoir. Un commissaire qui s'acquitte régulièrement de ses tâches est considéré par le ministre comme un fonctionnaire zélé. En se posant en interlocuteur privilégié, sinon exclusif, du ministère, le commissaire consolide ses positions locales. Il est intéressant à cet égard de repérer, dans la

(23) AN, FlcIII, Saône-et-Loire (8), Rapport du commissaire du Directoire exécutif près le tribunal civil de Chalon-sur-Saône, Carnot (prairial an VII). 
mesure du possible, les départements où le Ministère a mobilisé des réseaux d'informateurs officieux, à l'image de ces « commissaires aux barrières » dépêchés par Merlin en l'an VI, dont les rapports ont été publiés par J.-R. Suratteau (24), ou des «canaris», informateurs confidentiels de François de Neufchâteau en l'an VII, dont le réseau double la structure officielle (25). Le fait de ne pas répondre du tout aux sollicitations du Ministère peut être un moyen de ne pas trop attirer l'attention du niveau hiérarchique supérieur sur un département qui parvient à préserver ses équilibres internes. Mais ce peut être aussi le symptôme d'un malaise profond face aux exigences administratives d'un État moderne. À cet égard, la médiation personnelle ne suffit pas, le recours à l'écrit s'avère indispensable et, à travers lui, une compétence technique et juridique acquise. La formation intellectuelle de la majeure partie des commissaires est identique à celle des administrateurs et des députés, les fonctions étant bien souvent interchangeables; professions juridiques, expérience acquise dans la gestion des affaires locales, rudiments de fiscalité publique, techniques du renseignement.

La qualité rédactionnelle des rapports, un style clair et concis, la compréhension de la nature du renseignement demandé, sont des moyens d'évaluation de cette compétence administrative, dont l'apprentissage se fait massivement au contact du terrain. Les formulaires diffusés par le ministère, à titre de modèles, sont de plus en plus standardisés au fil de la période, pour arriver à la fin du Directoire à la confection de codes qui sont autant de petits manuels de droit administratif (26).

Mais cette uniformisation, et cette spécialisation croissantes, indispensables à la rationalisation du système administratif (27), ne constituent-elles pas un autre aspect du filtre socioculturel précédemment évoqué ? En utilisant un rapport standardisé, le commissaire gagne certes du temps, et l'information devient plus fluide (au niveau de l'enregistrement comme au niveau du dépouillement), mais, par la reproduction d'un modèle, n'y a-t-il pas le risque de fournir un document conforme à l'attente officielle, plutôt que le produit d'une observation authentique? Mais comment contrôler l'authenticité autrement que dans les conditions de production des témoignages qui prétendent en rendre compte?

(24) Jean-René SURATTEAU, Les élections de l'an VI et le «coup d'État» du 22 floréal (11 mai 1798), Paris, Les Belles-Lettres, 1971.

(25) Philippe BOURDIN, Le Puy-de-Dôme sous le Directoire; vie politique et esprit public, ClermontFerrand, Mémoires de l'Académie des Sciences, Belles Lettres et arts, 1991; Bernard GAINOT, 1799, un nouveau jacobinisme ? La démocratie représentative, une alternative à brumaire?, Paris, Éd. du C.T.H.S., 2001.

(26) Alexandre BALLY, Gabriel Gabet (1763-1853); du praticien de la Révolution au théoricien utopiste $d u X I X^{e}$ siècle, mémoire de maîtrise soutenu à l'Université de Bourgogne, sous la direction de Serge Wolikow. Gabriel Gabet, commissaire municipal de Dijon, est l'auteur d'un Code perpétuel ou manuel pratique à l'usage des commissaires auprès des administrations municipales, qui est reproduit en annexe du mémoire.

(27) Max WEBER, Économiè et Société, Paris, Plon, 1971. 
La prise de décision est inscrite dans les stratégies de pouvoir.

Trois niveaux d'analyse doivent être préalablement distingués.

-Tout d'abord le niveau local; c'est la configuration des relations entretenues par le commissaire avec des individus isolés, ou des groupes d'individus, ou des institutions, à finalité privée (famille, amis), semi-privée (sociabilité), publique (fréquence des contacts avec les autres commissaires, les administrateurs, les autres fonctionnaires). Cette configuration peut apparaître, notamment, dans l'analyse des conflits locaux, qui mettent aux prises les autorités constituées et la population locale. Prenons un exemple particulier: la commune de Tancon, dans le sud du département de la Saône-et-Loire, est un centre de résistance catholique, animé par un prêtre réfractaire, Vincent. Des rassemblements, taxés de «fanatiques » par les autorités de la municipalité de canton, Châteauneuf, se forment au début de l'an V. En nivôse an V (janvier 1797), le juge de paix de Châteauneuf, Claude Déal, frère de l'ancien maire de l'an II Antoine Déal, lance un mandat d'arrêt contre Vincent. Le chef-lieu fut en 1793 un centre actif de déchristianisation, et a conservé la réputation d'être «une vraie sentine révolutionnaire». Le personnel administratif de la période terroriste, installé par Javogues, a partiellement été remis en place au début du Directoire par le représentant du peuple Jacques Reverchon. La brigade de gendarmerie procède à l'arrestation de Vincent, mais rencontre une forte opposition de la population de Tancon, qui délivre le prisonnier. Le commissaire central du département, Etienne Rubat, un ami de Reverchon, demande une réquisition de la force armée au général Pille, qui commande la XVIII ${ }^{\mathrm{e}}$ division militaire à Dijon. Ce dernier donne un ordre de réquisition au général de brigade Gilbert Prudon, un militaire jacobin, pour commander les troupes dépêchées sur place par la division militaire, leur adjoindre vingt-quatre gendarmes originaires de Chalon-sur-Saône, et pour lever des colonnes mobiles parmi les gardes nationaux de Paray, CharoUes, Marcigny et La Clayette. Cette mobilisation militaire entraîne l'occupation par la force armée des communes de Tancon et Saint-Martin de Lixy (28).

Nous voyons avec cet exemple un règlement assez fréquent des conflits locaux; oppositions locales, sur-déterminées par l'expérience révolutionnaire, résistances communautaires, interaction des diverses autorités pour le retour au calme, qui ne peut s'opérer que par le recours à la force armée.

- Deuxième niveau d'analyse ; la stratégie de pouvoir proprement dite. Tout un réseau se mobilise en vue de la prise de décision.

Les décisions elles-mêmes se prennent au niveau central. C'est le processus de révocation/nomination, pour lequel interviennent les « recom-

(28) AN, FlbII, Saône-et-Loire, 1 (Affaires communales), et Archives départementales de Saône-etLoire, 5L13 (Procédure contre Laurent Dutreve et autres particuliers de Tancon). 
mandations », interférant avec le travail préparatoire des bureaux. Mais la «recommandation», d'un député le plus souvent, d'un conseiller du ministre ou d'un directeur parfois, quand la députation n'est pas «sûre », est largement le produit de rapports de forces locaux. Le commissaire central informe directement les bureaux, correspond avec tel ou tel député qui a l'oreille du Ministre, peut même se déplacer à Paris, muni de la liste des personnes à révoquer ou à placer. Tout dépend alors de la crédibilité du dit commissaire auprès du ministre, et c'est alors que la nature des comptes rendus administratifs qui lui sont parvenus joue un rôle déterminant.

Les exemples sont légion (29). Néanmoins, certaines périodes sont plus particulièrement propices au phénomène. Jacques Godechot avait relevé l'importance des mouvements consécutifs aux lendemains de fructidor an $\mathrm{V}$; mais il pensait que le second Directoire avait été marqué par une certaine stabilité :

«Après le coup d'État du 18 fructidor an V, il y eut, de nouveau, de nombreuses révocations, puis les deux dernières années du Directoire furent sensiblement plus calmes ; les administrations départementales, plus républicaines, se montrèrent désormais plus soumises. » (30)

Même si on ne prend en compte que les administrateurs élus, cette appréciation est discutable; pour les commissaires, elle est inexacte. Fructidor a bien souvent traduit, sur le plan local, la victoire, obtenue après d'âpres luttes, du commissaire du pouvoir exécutif sur les administrateurs élus. Mais, au cours des deux années suivantes, la volonté du Directoire de renforcer son contrôle sur les commissaires, au détriment de la latitude qui leur était précédemment laissée en tant qu'acteurs du jeu politique local, se traduisit par des mouvements très importants, surtout à la veille d'élections à l'issue incertaine. L'an VII fut particulièrement agité. Il y eut même une déstabilisation complète des deux tiers des administrations départementales; furent révoqués des commissaires qui étaient en poste depuis les débuts du Directoire. Ce ne furent pas les seules interventions de l'exécutif qui étaient responsables de cet état de fait. Le Corps législatif, humilié par les empiétements du second Directoire, prit sa revanche au lendemain des journées des 29 et 30 prairial an VII et sa cible privilégiée fut ces agents zélés que sont les commissaires installés, ou confortés quelques mois auparavant pour contrôler les élections (31).

Un seul exemple, pour illustrer notre propos (32) : dans le département de la Meuse, à Verdun, en l'an VII, Tocquot, ancien législateur et président

(29) Nous renvoyons, pour certains d'entre eux, à notre article paru dans la revue du « laboratoire des collectivités locales» de la faculté de droit de l'Université d'Orléans, sous la direction de Michel Pertué ; Bernard GAINOT, « Rivalités locales et nationalisation des enjeux ; notables, réseaux et partis sous le Directoire » (1997).

(30) Jacques GODECHOT, Les institutions..., op. cit., pp. 51-52.

(31) Bernard GAINOT, 1799, un nouveau jacobinisme..., op. cit.

(32) AN, AF III 248. 
de l'administration centrale, entretient une correspondance suivie avec le ministre François de Neufchâteau à la veille des élections. Ses analyses recoupent et confortent celles du commissaire central, Jean-Antoine Henriot, installé depuis les débuts du Directoire. Il s'agit de contrer l'organisation du "groupe anarchiste », dont l'intermédiaire à Paris est l'ancien conventionnel Robert Pons. La défaite électorale des « républicains gouvernementaux », imputée à une manœuvre conjointe des «anarchistes» de Pons et des «royalistes » dirigés par Jean-Baptiste Harmand, entraîne la révocation de Henriot, remplacé par le frère de Pons, Clément Pons. Le champ fluctuant des rapports de forces locaux couvre aussi bien les assemblées électorales du département que les interventions auprès du pouvoir central. Tocquot présente ainsi Pierre Arnoud, l'intermédiaire parisien d'Harmand :

« on présente Arnoud comme tout-puissant au Directoire, il promet des places de tout genre et c'est surtout dans la prochaine organisation forestière... »

Quant à Pons, le 30 prairial renforce ses positions, puisqu'il parvient à faire nommer son frère au poste de commissaire central.

-Le troisième niveau d'analyse est celui de l'évaluation de l'action proprement dite.

Évaluer les conséquences d'un acte administratif ne peut se faire indépendamment des formes par lesquelles cet acte est énoncé et annoncé. Il faut envisager la production administrative comme production de textes normatifs, mais aussi performatifs, c'est-à-dire non seulement régulatrice des équilibres sociaux, mais également créatrice d'une structure sociale envisagée sous l'angle des relations de pouvoir (33). Il reste à mesurer la pertinence de ce type d'approche, surtout utilisée à propos de textes de philosophie politique (fortement individualisés), ou de pièces de la rhétorique d'assemblées (fortement contextualisées) (34). Qu'en est-il des circulaires administratives, textes où le simple caractère énonciatif semble épuiser toute autre velléité interprétative, donc découpler radicalement le moment de l'action et celui de la décision?

En conclusion, nous souhaiterions replacer ces quelques pistes de recherches dans une perspective comparatiste. Comparaison avec d'autres espaces et d'autres temps : mais les structures sociales sont-elles radicale-

(33) J.-L. AUSTIN, Quand dire, c'est faire (traduction de Gilles Lane), Paris, Éditions du Seuil, 1970 ; Jacques GUILHAUMOU, La langue politique et la Révolution française, Paris, Méridiens/Klincksieck, 1989.

(34) Quentin SKINNER, Les fondements de la pensée politique moderne, (traduction de Jérôme Grossman et Jean-Yves Pouilloux), Paris, Albin Michel, 2001. 
ment autres? J'emprunte la citation suivante à un historien napolitain, à propos de la Sicile des années 1860 :

«Comme en bien d'autres circonstances au cours des XIX et $\mathrm{XX}^{\mathrm{e}}$ siècles, les individus interviennent dans la Grande Histoire en fonction de leurs intérêts propres, pour affronter la petite histoire des bourgs, des villages, des familles. La violence joue alors le rôle d'accoucheuse, sinon d'une nouvelle civilisation, du moins de nouveaux équilibres; le processus révolutionnaire définit les conflits politiques et sociaux; il permet d'exercer des vengeances privées, d'accéder à la richesse et au pouvoir. » (35)

Il suffit de remplacer «violence » par «gestion des affaires locales » et nous avons, mutatis mutandis, la position des commissaires centraux, quoique la richesse ne semble guère acquise, si l'on en croit les plaintes récurrentes concernant leur traitement. Mais c'est un autre aspect (important) du problème. Comparaison également avec d'autres institutions, avant tout l'institution préfectorale (36) : ce qui se joue à ce niveau, c'est le rôle de la médiation dans la redéfinition du lien social, alors que la Révolution a voulu justement supprimer les corps intermédiaires issus de l'Ancien Régime. Ce faisant, les rapports sociaux se recomposent. L'échelon administratif n'est pas une simple courroie de transmission de l'État : si le commissaire du Directoire exécutif n'est pas encore ce notable, dont Lucien Bonaparte esquissera les fonctions et les missions dans la circulaire de ventôse an VIII sur l'institution préfectorale, il n'en joue pas moins, à son niveau, le rôle de proto-notable, tant par les liens qu'il tisse avec son environnement, que par ses initiatives en matière économique et pour la création d'un espace public.

\author{
Bernard GAINOT \\ Institut d'Histoire de la Révolution française \\ Université Paris I Panthéon-Sorbonne \\ 69, avenue Danièle Casanova \\ 94200 Ivry-sur-Seine
}

(35) Salvatore LUPO, Histoire de la mafia, des origines à nos jours (traduction de Jean-Claude Zancarini), Paris, Flammarion, 1999, p. 57.

(36) Éric BRIAN, La mesure de l'État. Administrateurs et géomètres au XVIII siècle, Paris, Albin Michel, 1994 ; voir également Alain COTTEREAU, « Espace public et capacité de juger. La stabilisation d'un espace public en France aux lendemains de la Révolution » dans Raisons pratiques, 3 (1992), pp. 239-273. 